\title{
Axillary artery occlusion as a presenting feature of Crohn's disease
}

\author{
R.A. Sheehan-Dare, M.J.D. Goodfield, P.D. Wilson ${ }^{1}$ and N.R.Rowell \\ Departments of Dermatology, Leeds General Infirmary, Leeds LS1 3EX and ' 'Grimsby District Hospital, Grimsby, \\ UK.
}

\begin{abstract}
Summary: A 20 year old Caucasian woman with Crohn's disease is described in whom axillary artery occlusion due to arteritis dominated the clinical picture. This case demonstrates that isolated large vessel arteritis can be an unusual feature of Crohn's disease. Crohn's disease should be considered in any patient presenting with a large vessel arteritis.
\end{abstract}

\section{Introduction}

Inflammatory bowel disease may be associated with a variety of extra-intestinal features. Both Crohn's disease and ulcerative colitis have been associated with a widespread arteritis indistinguishable from Takayasu's arteritis. ${ }^{1-7}$ We present a patient with Crohn's disease in whom an isolated large vessel obstruction due to arteritis dominated the clinical picture.

\section{Case report}

A 20 year old Caucasian woman presented with a 4-week history of erythema nodosum on the legs which settled with conservative measures. This was followed several weeks later by pain and paraesthesiae in the right arm, forearm and hand. In addition there were joint pains and a recurrence of the erythema nodosum. The patient was receiving no medication, including oral contraceptives.

Examination revealed a palpable tender right axillary artery with absence of the distal pulses and splinter haemorrhages in the fingers of the right hand. In addition there was a symmetrical large joint arthritis involving the knees and wrists. Haemoglobin was $11.4 \mathrm{~g} / \mathrm{dl}$, platelets $459 \times 10^{9} / 1$ and erythrocyte sedimentation rate elevated at $80 \mathrm{~mm} / \mathrm{h}$. Serum biochemistry and coagulation studies were normal.

The erythema nodosum and arthritis resolved over several weeks with symptomatic measures but the radial and brachial pulses in the right arm remained absent. The patient subsequently developed mild

Correspondence: R.A. Sheehan-Dare, M.B., Ch.B., M.R.C.P.

Accepted: 16 May 1989 diarrhoea associated with weight loss. Investigations at this time revealed a haemoglobin of $10.3 \mathrm{~g} / \mathrm{dl}$, white cells $12 \times 10^{9} / 1$ with a normal differential count, platelets $522 \times 10^{9} / 1$ and an erythrocyte sedimentation rate of $68 \mathrm{~mm} / \mathrm{h}$. Coagulation studies including tests for the lupus anticoagulant were entirely normal. Antinuclear antibody and rheumatoid factor were not detected.

Arteriography demonstrated a generalized reduc tion in calibre of the right subclavian artery distal to the thyro-cervical trunk (Figure 1) with tapering of the

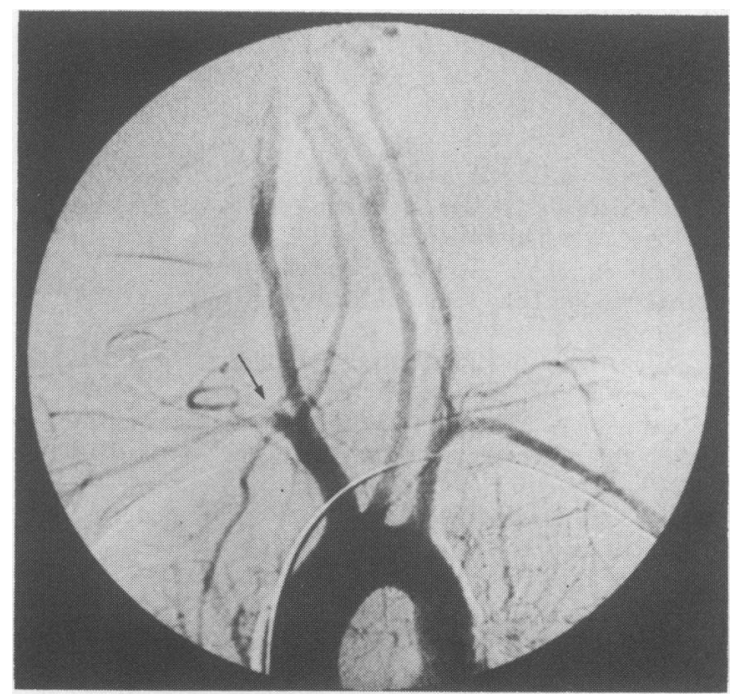

Figure 1 Arch aortogram showing concentric narrowing of the right subclavian artery distal to the thyro-cervical trunk (arrow). 
proximal axillary artery leading to a complete occlusion. However, there was already a good collateral circulation. The aorta and the remainder of its branches were of normal calibre. The appearances were of limited arteritis with a secondary vascular occlusion. A double contrast barium enema revealed features typical of Crohn's disease. There was widespread mucosal irregularity and aphthoid ulceration of the colon with skip lesions and sparing of the rectal mucosa (Figure 2). In addition there was involvement of the terminal ileum (Figure 3). Stool cultures revealed no pathogenic organisms. A diagnosis of occlusive axillary arteritis due to Crohn's disease was made.

The Crohn's disease responded to treatment with oral prednisolone $40 \mathrm{mg}$ daily, reducing to a maintenance dose. The absence of radial and brachial pulses in the right arm persisted despite corticosteroid therapy.

\section{Discussion}

This case demonstrates that isolated large artery occlusion due to limited arteritis can be an unusual

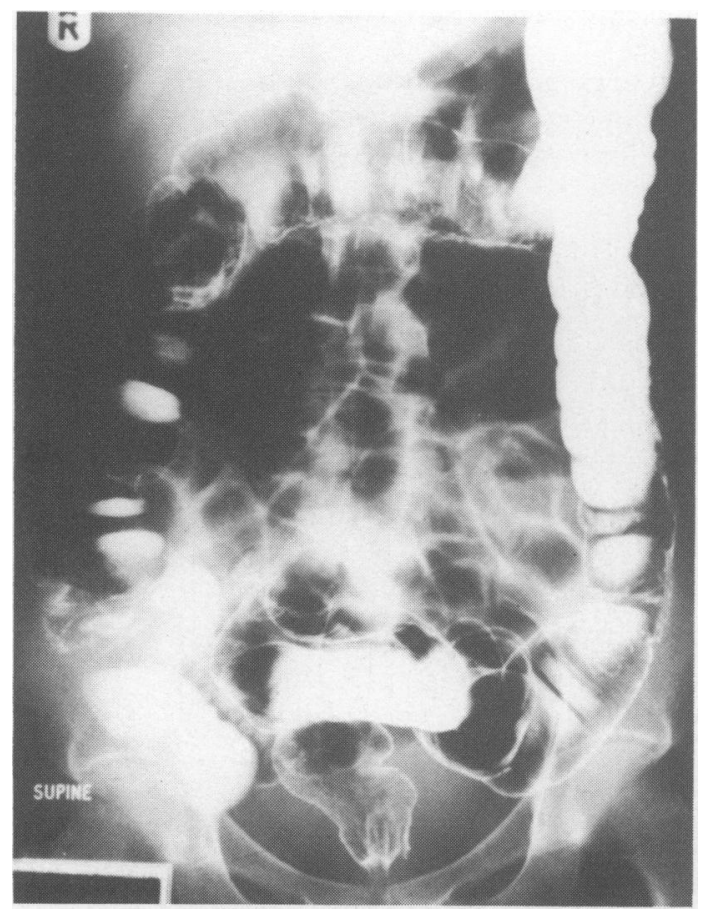

Figure 2 Double contrast barium enema showing extensive areas of mucosal irregularity of the colon separated by mucosa of normal appearance. presenting feature of Crohn's disease and may dominate the clinical picture. Large vessel arteritis occurring in association with inflammatory bowel disease has been reported infrequently. An association with both Crohn's disease ${ }^{1-4}$ and ulcerative colitis ${ }^{5-7}$ has been reported. In these reports, the large vessel arteritis has been in the form of multiple widespread lesions with involvement of the aorta indistinguishable clinically $^{1-7}$ and histologically ${ }^{1}$ from Takayasu's disease. Limited large vessel arteritis has not previously been described.

A large joint symmetrical arthritis and erythema nodosum were associated features in our patient at presentation with arteritis. Arthritis was present at the time of presentation of arteritis in only one of the four cases previously reported. ${ }^{4}$ Erythema nodosum was observed in two of these cases at an earlier stage in their disease but not at the time of presentation with arteritis. ${ }^{1,2} \mathrm{~A}$ further patient developed an ulcerative nodular vasculitis with panniculitis on the thighs and calves at the time of presentation with arteritis but the appearances were not typical of erythema nodosum. ${ }^{3}$

It would appear that a range of arterial lesions can occur in association with inflammatory bowel disease. We suggest that large vessel arteritis, including aor-

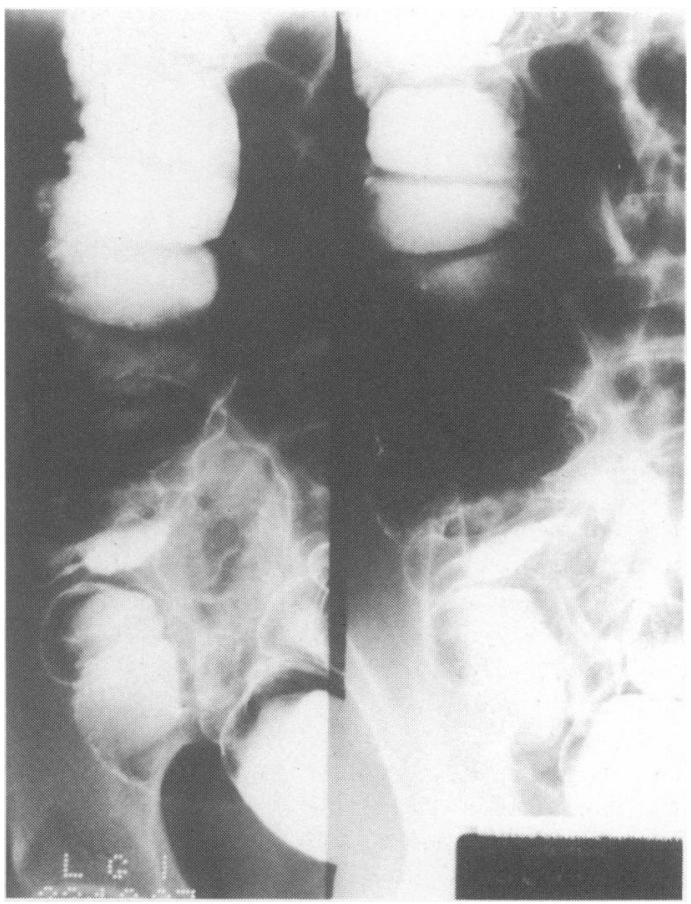

Figure 3 Double contrast barium enema showing mucosal irregularity with aphthoid ulceration in the ascending colon and involvement of the terminal ileum. 
titis, should be considered an extra-intestinal manifestation of inflammatory bowel disease rather than two separate disorders occurring simultaneously.

In most previous reports inflammatory bowel disease has been the major presenting feature and has preceded or in some cases coincided with the develop-

\section{References}

1. Owyang, C., Miller, L.J., Lie, J.T. \& Fleming, C.R. Takayasu's arteritis in Crohn's disease. Gastroenterology 1979, 76: 825-828.

2. Yassinger, S., Adelman, R., Cantor, D., Halstead, C.H. \& Bolt, R.J. Association of inflammatory bowel disease and large vascular lesions. Gastroenterology 1976, 71: 844-846.

3. Friedman, C.J. \& Tegtmeyer, C.J. Crohn's disease associated with Takayasu's arteritis. Dig Dis Sci 1979, 24: 954-958.

4. Lenhoff, S.J. \& Mee, A.S. Crohn's disease of the colon with Takayasu's arteritis. Postgrad Med J 1982, 58: 386-389. ment of arteritis. In our patient however, the arteritis preceded symptoms of Crohn's disease. We therefore suggest that underlying inflammatory bowel disease should be considered in any patient presenting with a large vessel arteritis.

5. Chapman, R., Dawe, C., Whorwell, P.J. \& Wright, R. $\vec{\circ}$ Ulcerative colitis in association with Takayasu's disease. Am J Dig Dis 1978, 23: 660-662.

6. Soloway, M., Moir, T.W., Linton, D.S. Takayasu's arteritis. Report of a case with unusual findings. $\mathrm{Am}$ go Cardiol 1970, 25: 258-263.

7. Achar, K.N. \& AL-Nakib, B. Takayasu's arteritis and ulcerative colitis. Am J Gastroenterol 1986, 81: 1215-1217. 